\title{
Prognosen als Grundlage der Vertriebsplanung
}

Wir kennen ca. 30 grundsätzliche Methoden, die helfen, den zukünftigen Vertriebserfolg vorauszusagen, viele davon mit Unterarten. Wir finden kreative Verfahren, Verfahren auf Basis von Expertenwissen, solche, die einfache Mathematik benötigen, wie sie jedes Tabellenkalkulationsprogramm beherrscht, und ökonometrische Verfahren, die Spezialisten vorbehalten bleiben. In diesem Essential beschränken wir uns auf Methoden, die sich erklären lassen, ohne dass ein Studium statistischer Methodenlehre vorausgesetzt wird. Ziel ist, dass Vertriebscontroller - oder wer auch immer im Unternehmen dafür verantwortlich sein mag - Vertriebsprognosen erstellen kann.

Bevor wir damit beginnen, soll erläutert werden, wozu Prognosen taugen, was sie also zu leisten imstande sind, und wie mit Ihnen umzugehen ist. Dieser korrekte Umgang mit Prognosen ist keine Selbstverständlichkeit, denn nur allzu oft werden im unternehmerischen Umfeld „Planung“ und „Forecast“ verwechselt. Prognosen sind Zukunftsprojektionen auf Basis nachvollziehbarer Modelle und Annahmen. Die Ergebnisse sind eine Vermutung, wie die Zukunft wahrscheinlich ihren Lauf nehmen wird. Auf Basis dieser Vermutung wird dann geplant, was nichts anderes bedeutet, als dass die dem Unternehmen zur Verfügung stehenden Ressourcen allokiert werden. 